\title{
Phenotype-based screening of selected mangrove methanolic crude extracts with anti-melanogenic activity using zebrafish (Danio rerio) as a model
}

\author{
Mark Andre C. Grisola, Rolly G. Fuentes* \\ Division of Natural Sciences and Mathematics, University of the Philippines Visayas Tacloban College, \\ 6500 Tacloban City, Philippines
}

*Corresponding author, e-mail: rgfuentes@up.edu.ph

Received 24 Sep 2016

Accepted 10 Jun 2017

\begin{abstract}
Novel skin hypopigmenting agents are important due to the unwanted side effects of existing therapies and treatments. In this study, the melanogenic effects of crude extracts of selected mangroves, namely, Avicennia marina, Excoecaria agallocha, Rhizophora apiculata, and Sonneratia alba were screened using zebrafish as a model organism. During the screening process, $50 \mu \mathrm{g} / \mathrm{ml}$ and $100 \mu \mathrm{g} / \mathrm{ml}$ concentrations of the extracts were applied to the zebrafish embryos at $9 \mathrm{~h}$ post fertilization and effects were observed at $55 \mathrm{~h}$ post fertilization. Among the extracts, $S$. alba bark extract displayed the best results in terms of its effect on melanogenesis and toxicity. It was further investigated and was found to have a dose-dependent effect on the pigmentation of embryos. Higher dosages of $S$. alba bark extract such as $400 \mu \mathrm{g} / \mathrm{ml}$ and $500 \mu \mathrm{g} / \mathrm{ml}$ caused profound effects on embryo melanogenesis. IMAGEJ analysis revealed that higher dosages remarkably decreased the ocular melanin content of the embryos. Results suggest that $S$. alba is a potent source of compounds with anti-melanogenic properties.
\end{abstract}

KEYWORDS: melanin, embryo

\section{INTRODUCTION}

Skin pigmentation is attributed to melanin, which is a darkening pigment synthesized and secreted by melanocytes found in the basal layer of the epidermis through a process called melanogenesis $^{1}$. Melanogenesis is initiated and catalysed by the key enzyme tyrosinase ${ }^{1,2}$. Melanin is important in the protection of human skin from the harmful effects of UV light radiation and in the scavenging of free radicals generated within the cytoplasm. However, excessive production and abnormal distribution of melanin may cause serious aesthetic problems particularly hyperpigmentation disorders such as melasma, Riehl's melanosis, and postinflammatory hyperpigmentation ${ }^{3}$. Hyperpigmentation or skin darkening is one of the most prevalent dermal conditions which is particularly resistant to treatment. Hyperpigmentation remains a clinical management challenge for dermatologists despite the availability of multiple treatments ${ }^{4}$. Considered as the gold standard among commonly available topical treatments, hydroquinone causes adverse effects such as skin irritation, exogenous ochronosis, and permanent depigmentation ${ }^{5}$. These unwanted side effects have led researchers to seek for agents from plants which are considered safer alternative ${ }^{6}$. Most of the identified plants considered to be potential sources of melanogenesis inhibitors were screened for their tyrosine inhibitory activity. Some of these screened plants are Vaccinium bracteatum ${ }^{7}$ and Panax ginseng ${ }^{8}$. Screening of 67 tropical plants identified Entada africana, Portulaca pilosa, Prosopis africana, Stryphnodendron barbatimao, and Cariniana brasiliensis to exhibit strong tyrosine inhibitory activity ${ }^{9}$. A study using a mouse model reported that the plant Artemisia capillaris exhibited antipigmentation activity and the compound 4,5dicaffeoylquinic acid was identified to be the active compound ${ }^{10}$.

A recent study has validated the use of zebrafish (Danio rerio) as a whole-animal model organism for the study of melanogenesis ${ }^{11}$. This study has also identified several advantages of using zebrafish including the rapidity, cost-effectiveness, and physiological relevance. Zebrafish was also used to further establish the whitening effects and toxicity of the compound octaphlorethol isolated from an alga Ishige foliacea ${ }^{12}$. In this study, zebrafish assay model was used to screen for the anti-melanogenic 
activities of some mangrove plants. The screening study led to the identification of $S$. alba with potent anti-melanogenic activity which supports the previous study ${ }^{13}$ on tyrosinase inhibitory activity of the extract from S. alba. To the best of our knowledge, this is the first report of a phenotypic evidence of the anti-melanogenic activity of $S$. alba using an animal model.

\section{MATERIALS AND METHODS}

Origin and maintenance of parental zebrafish

Adult zebrafish were obtained from a commercial dealer in Tacloban City, Philippines. Zebrafish were maintained following a standard protocol given in Ref. 14. They were kept in an aquarium equipped with water aerator and maintained at a cycle of $14 \mathrm{~h} \mathrm{light}$ and $10 \mathrm{~h}$ dark in order to simulate the optimal natural breeding conditions. They were fed twice a day with flake food supplement, once in the morning and once in the afternoon.

\section{Preparation of methanolic extracts}

Various parts of selected mangrove species, namely, Avicennia marina, Excoecaria agallocha, Rhizophora apiculata, and Sonneratia alba were collected from Sitio Mahulos Brgy. Ponong Carigara, Leyte, Philippines. Voucher specimens were kept at the Division of Natural Sciences and Mathematics, University of the Philippines Visayas Tacloban College. Their identifications were verified using a field guide ${ }^{15}$. The collected parts such as barks, leaves, stems, roots, flowers, and fruits were washed thoroughly with water and were air-dried. One hundred grams of each plant material was homogenized with $250 \mathrm{ml}$ methanol. Mixtures were left overnight, filtered through a Whatman filter paper and solvents were removed in vacuo (Buchi). Dry extracts were kept refrigerated at $4{ }^{\circ} \mathrm{C}$ until analysis.

\section{Phenotype-based evaluation using zebrafish}

The assay using the zebrafish model was conducted using the method described previously ${ }^{11}$. Kojic acid, a known whitening agent and a tyrosinase inhibitor, was used as a positive control. Spawning was done by placing male and female zebrafish in a 11 breeding tank and was induced in the morning as soon as the light cycle started. Embryos were collected, washed and examined under a stereomicroscope in order to remove any unfertilized eggs. Then the embryos were transferred to a Petri dish filled with embryonic medium and incubated at $28.5^{\circ} \mathrm{C}$. Synchronized embryos were collected and randomly transferred to a 24-well plate containing embryonic medium, each well having ten embryos. Three wells were allotted for each concentration of tyrosinase from the extract which summed up to 30 eggs per concentration per trial. The embryos were treated with plant extracts (dissolved in $0.1 \%$ DMSO) from $9 \mathrm{~h}$ post fertilization (hpf) to $55 \mathrm{hpf}$. To ensure the even distribution of the extract in all wells, occasional stirring as well as replacement of the medium with extract were done daily. At $55 \mathrm{hpf}$, the effects of the extract on the pigmentation of the living embryos only were observed under the microscope and were photographed. The effects on the pigmentation of zebrafish were scored as follows: $<10 \%$ as none or mild, $10-49 \%$ as moderate, and $>50 \%$ as profound ${ }^{11}$.

\section{Estimation of ocular melanin content}

Melanin content of the treated embryos was estimated using IMAGEJ analysis software ${ }^{16}$. Ten representative embryos from each dosage group including the control and kojic acid were randomly photographed. The area of the eyes, which has the greatest intensity, was outlined and the integrated density was calculated to quantify the melanin content. Integrated density is the product of the outlined area and its mean grey value. Higher value of integrated density means darker eyes.

\section{Data analysis}

The melanin content of the representative embryos was evaluated statistically using One-way Analysis of Variance. The post hoc test Tukey's Honest Significant Difference was performed after ANOVA to compare all possible pair of means and to identify which pair is significantly different from each other.

\section{RESULTS AND DISCUSSION}

Various parts of selected mangrove species were investigated for their toxicity and effects on the melanogenesis of zebrafish embryos. Some extracts caused death of the embryos (Table 1). Only R. apiculata bark, leaf, and stem extracts and S. alba bark extract were not lethal to the embryos for both concentrations.

During the screening study, all mangrove extracts only showed none or mild $(<10 \%)$ inhibitory effects on the melanogenesis of the embryos (Fig. 1). However, S. alba bark extract displayed the best results. One convincing evidence was that the eyes and the melanocytes, occupying an area which covered from the eyes to the anterior extent of the yolk sac, were generally lighter in embryos treated 
Table 1 Toxicity of selected mangrove samples to the zebrafish embryos.

\begin{tabular}{|c|c|c|c|}
\hline \multicolumn{2}{|l|}{ Treatment } & Conc. $(\mu \mathrm{g} / \mathrm{ml})$ & Embryo $^{a}$ \\
\hline \multicolumn{2}{|c|}{ Control (0.1\% DMSO) } & & 0 \\
\hline \multicolumn{2}{|c|}{ Kojic acid $(20 \mathrm{mM})$} & & 0 \\
\hline \multirow[t]{10}{*}{ A. marina } & Bark & 50 & $14 / 30$ \\
\hline & & 100 & $16 / 30$ \\
\hline & Leaf & 50 & $20 / 30$ \\
\hline & & 100 & $30 / 30$ \\
\hline & Stem & 50 & $16 / 30$ \\
\hline & & 100 & $30 / 30$ \\
\hline & Flower & 50 & $1 / 30$ \\
\hline & & 100 & $12 / 30$ \\
\hline & Fruit & 50 & 0 \\
\hline & & 100 & $1 / 30$ \\
\hline \multirow[t]{6}{*}{ E. agallocha } & Bark & 50 & $30 / 30$ \\
\hline & & 100 & $30 / 30$ \\
\hline & Leaf & 50 & $30 / 30$ \\
\hline & & 100 & $27 / 30$ \\
\hline & Stem & 50 & $30 / 30$ \\
\hline & & 100 & $30 / 30$ \\
\hline \multirow[t]{8}{*}{ R. apiculata } & Bark & 50 & 0 \\
\hline & & 100 & 0 \\
\hline & Leaf & 50 & 0 \\
\hline & & 100 & 0 \\
\hline & Stem & 50 & 0 \\
\hline & & 100 & 0 \\
\hline & Root & 50 & $7 / 30$ \\
\hline & & 100 & $14 / 30$ \\
\hline \multirow[t]{4}{*}{ S. alba } & Bark & 50 & 0 \\
\hline & & 100 & 0 \\
\hline & Leaf & 50 & $30 / 30$ \\
\hline & & 100 & $30 / 30$ \\
\hline
\end{tabular}

a death/total
Table 2 Effects of Sonneratia alba bark extract on the pigmentation of embryos.

\begin{tabular}{lcccc}
\hline Treatment & Conc. $^{\text {a }}$ & None/Mild & Mod. $^{*}$ & Profound $^{*}$ \\
\hline Control $^{\dagger}$ & & $30(100)$ & $0(0)$ & $0(0)$ \\
Kojic acid $^{*}$ & & $0(0)$ & $0(0)$ & $30(100)$ \\
S. alba bark & 500 & $0(0)$ & $0(0)$ & $30(100)$ \\
& 400 & $0(0)$ & $2(7)$ & $28(93)$ \\
& 300 & $13(43)$ & $15(5)$ & $2(7)$ \\
& 200 & $29(97)$ & $1(3)$ & $0(0)$ \\
& 100 & $30(100)$ & $0(0)$ & $0(0)$ \\
\hline
\end{tabular}

${ }^{\mathrm{a}} \mu \mathrm{g} / \mathrm{ml} ;{ }^{\dagger} 0.1 \% \mathrm{DMSO} ; 20 \mathrm{mM}$.

* The values in each column indicate the number of embryos that exhibited the respective criterion. Values inside the parenthesis are the corresponding percentages of the embryos that exhibited the respective criterion or effect. Data are representative of three independent experiments.

with $S$. alba bark extract. Besides, embryos treated with $50 \mu \mathrm{g} / \mathrm{ml} \mathrm{S}$. alba bark already exhibited mild melanogenic effects.

$S$. alba was further investigated to evaluate more precisely its anti-melanogenic property. Its bark extract showed a dose-dependent inhibitory effect on the melanogenesis of zebrafish. Higher dosages showed profound $(>50 \%)$ inhibition while lower dosages showed moderate and mild (1040\%) effects (Table 2; Fig. 2). At high concentration of the extracts $(400 \mu \mathrm{g} / \mathrm{ml}$ and $500 \mu \mathrm{g} / \mathrm{ml})$, a high number of cases of embryo exhibiting profound effects of the extract were observed. The embryos treated with higher dosages were generally

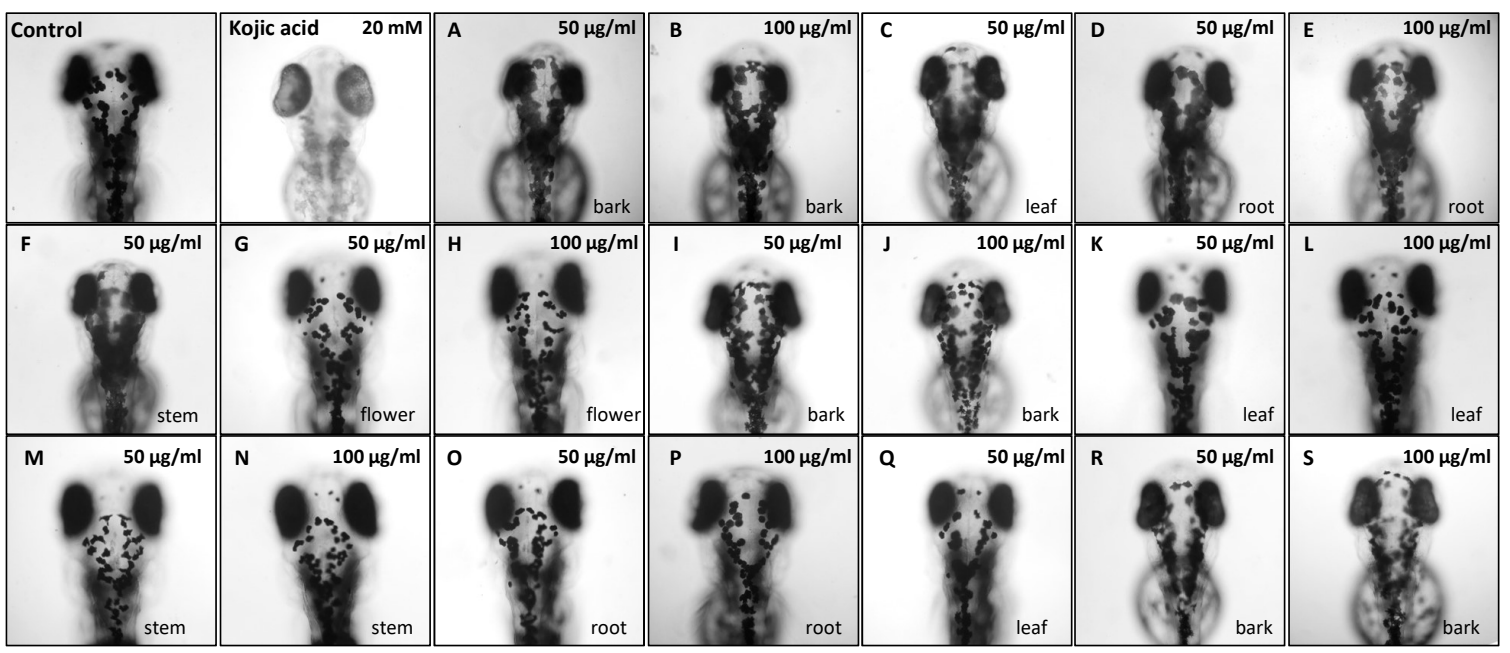

Fig. 1 Melanogenic effects on the zebrafish embryo pigmentation, viewed under the microscope, of selected mangrove extracts at concentrations $100 \mu \mathrm{g} / \mathrm{ml}$ and $50 \mu \mathrm{g} / \mathrm{ml}$; A-H: A. marina, I-P: R. apiculata, Q: E. agallocha, R-S: S. alba. 


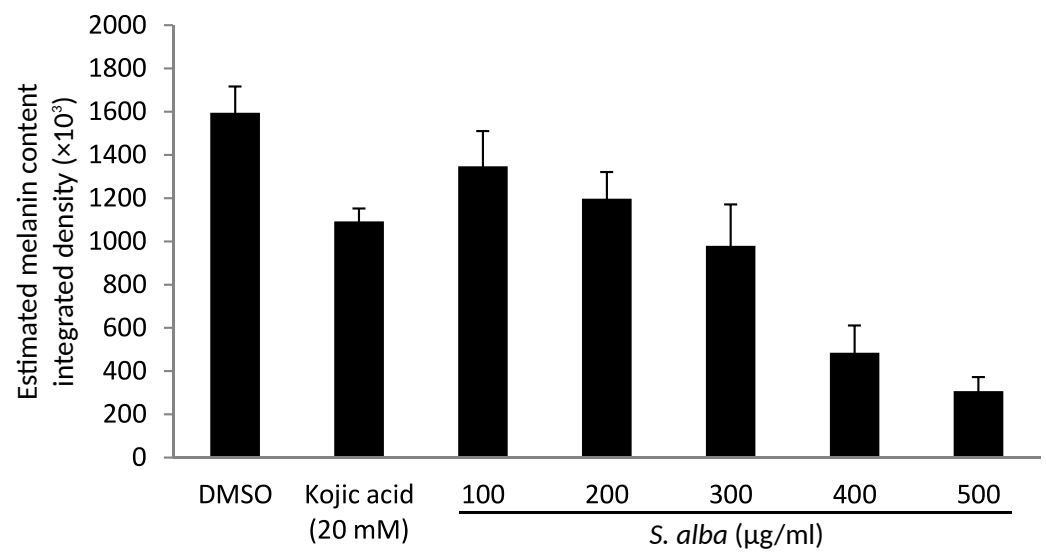

Fig. 2 Relative pigmentation intensity in eyes. Levels of eye pigmentation (black coloration intensity) of ten representative embryos per dosage group were quantified using IMAGEJ. The bar represents the mean $( \pm S D)$ integrated densities or estimated ocular melanin content of zebrafish embryos. Data are representative of three independent experiments.

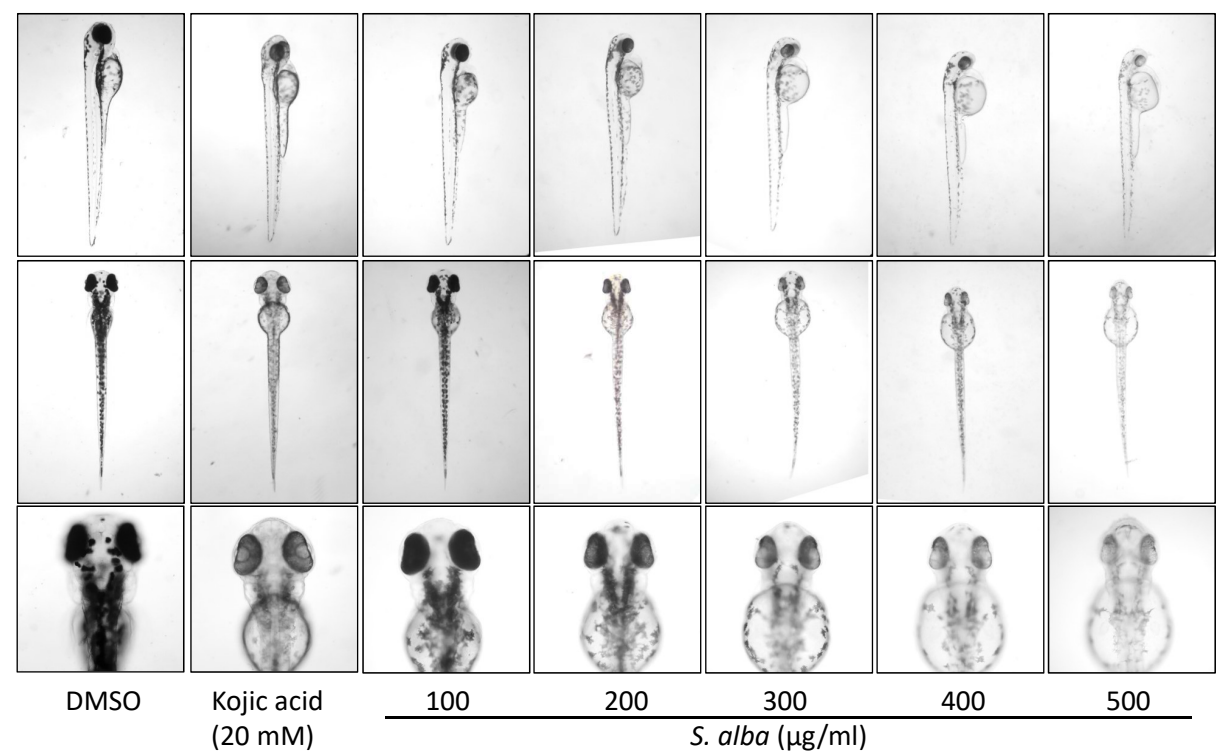

Fig. 3 Dose-dependent effect of $S$. alba on the pigmentation of zebrafish embryos. The effects of the extracts on the pigmentation were viewed under the microscope.

lighter compared to the embryos treated with lower dosages of $S$. alba bark extract. Furthermore, the effects of the extract on the pigmentation of the body and retina pigment epithelium were evident at higher dosages (Fig. 3). At higher dosages, the crude extract did not cause death (Table 2). Apparently, the $S$. alba bark extract showed inhibitory effect on the melanogenesis of zebrafish ochronosis embryos. Results of the IMAGEJ analysis revealed that as the concentration of the extract increased, the eye integrated density of the embryos remarkably decreased (Fig. 3). At highest concentration the pigment intensity was low as shown by the low integrated density value. The integrated density gives the estimated melanin content ${ }^{17}$. Thus the results suggest that $S$. alba extract decreased the ocular melanin content in zebrafish embryo.

There are three known mechanisms for the down-regulation of the tyrosinase activity ${ }^{18}$. The most common mechanism reported is the direct inhibition of tyrosinase catalytic activity through tyrosinase inhibitors. Plant based tyrosinase inhibitors have already been identified. Some of these are active compounds that are present in the se- 
lected mangroves. In this study, S. alba bark extract was shown to have a dose-dependent melanogenic inhibitory potential on zebrafish embryos. Profound effects were observed at higher dosages and the ocular melanin content remarkably decreased as the concentration of the extract was increased. The bark extract of $S$. alba were found to contain bioactive compounds particularly lupeol and hydrolysable tannins ${ }^{19,20}$. These two bioactive compounds have shown anti-tyrosinase activity in several studies in vitro. Lupeol, a lupane type triterpenoid, exhibited significant activity against tyrosinase enzyme ${ }^{21}$. It was also reported that $3,3^{\prime}$-di-O-methyl ellagic acid is present in the bark extract of $S$. alba ${ }^{22}$. The rind extract of pomegranate, which is rich in ellagic acid, exhibited inhibitory activity against mushroom tyrosinase in vitro ${ }^{23}$. The effect was even comparable to that of arbutin, a known hypopigmenting agent. The inhibitory activity on melanogenesis of ellagic acid has also been reported ${ }^{24}$. Furthermore, the bark extract of $S$. alba had been reported to exhibit high anti-tyrosinase activity ${ }^{13}$. The inhibitory activity was almost twice as high compared to that of green tea extract, a known skin whitener. Furthermore, the study attributed the high tyrosinase inhibitory effect to the high phenol content of $S$. alba bark extract. Thus the compounds discussed above can possibly contribute to the tyrosinase inhibitory activity in S. alba. Thus the inhibition of melanin content in the zebrafish embryo could be possibly due to the anti-tyrosinase inhibitory activities of the compounds present in the bark extract.

In conclusion, this study revealed that $S$. alba bark extract exhibited a dose-dependent inhibitory effect on the melanogenesis of zebrafish embryos. This is the first study to report the anti-melanogenic activity of the $S$. alba bark extract in an in vivo model. The results of this study support previous studies which suggest that $S$. alba is a potential source of compounds that could inhibit melanogenesis. Further investigation must be done to determine the inhibitory mechanism of $S$. alba bark extract.

Acknowledgements: This study was supported by the Small Budget In-House Research Grant through the Office of the Vice-Chancellor for Research and Extension, University of the Philippines Visayas.

\section{REFERENCES}

1. Gillbro J, Olsson M (2010) The melanogenesis and mechanisms of skin-lightening agents-existing and new approaches. Int J Cosmet Sci 33, 210-21.
2. Ebanks JP, Wickett RR, Boissy RE (2009) Mechanisms regulating skin pigmentation: The rise and fall of complexion coloration. Int J Mol Sci 10, 4066-87.

3. Jimbow K, Minamitsuji Y (2001) Topical therapies for melasma and disorders of hyperpigmentation. Dermatol Ther 14, 35-45.

4. Desai SR (2014) Hyperpigmentation therapy: a review. J Clin Aesthetic Dermatol 7(8), 13-7.

5. Shin JW, Park KC (2014) Current clinical use of depigmenting agents. Dermatol Sin 32, 205-10.

6. Zhu W, Gao J (2008) The use of botanical extracts as topical skin-lightening agents for the improvement of skin pigmentation disorders. $J$ Investig Dermatol Symp Proc 13, 20-4.

7. Kim M, Park J, Song K, Kim HG, Koh J-S, Boo YC (2012) Screening of plant extracts for human tyrosinase inhibiting effects. Int $J$ Cosmet Sci 34, 202-8.

8. Lee Y, Kim K-T, Kim SS, Hur J, Ha SK, Cho C-W, Choi SY (2014) Inhibitory effects of ginseng seed on melanin biosynthesis. Pharmacogn Mag 10, S272-5.

9. Baurin N, Arnoult E, Scior T, Do QT, Bernard P (2002) Preliminary screening of some tropical plants for anti-tyrosinase activity. $J$ Ethnopharmacol 82, 155-8.

10. Tabassum N, Lee J-H, Yim S-H, Batkhuu GJ, Jung DW, Williams DR (2016) Isolation of 4,5-O-dicaffeoylquinic acid as a pigmentation inhibitor occurring in Artemisia capillaris Thunberg and its validation in vivo. Evid Based Complement Altern Med 2016, 7823541.

11. Choi TY, Kim JH, Ko DH, Kim CH, Hwang JS, Ahn S, Kim SY, Kim CD, Lee JH, Yoon TJ (2007) Zebrafish as a new model for phenotype-based screening of melanogenic regulatory compounds. Pigment Cell Res 20, 120-7.

12. Kim KN, Yang HM, Kang SM, Ahn GN, Roh SW, Lee W, Kim DK, Jeon YJ (2015) Whitening effect of octaphlorethol A isolated from Ishige foliacea in an in vivo zebrafish model. J Microbiol Biotechnol 25, 448-51.

13. Suh S, Hwang J, Park M, Park HS, Lee TK (2014) Phenol content, antioxidant and tyrosinase inhibitory activity of mangrove plants in Micronesia. Asian Pac $J$ Trop Med 7, 531-5.

14. Westerfield M (2000) The Zebrafish Book. A Guide for the Laboratory Use of Zebrafish (Danio rerio), 4th edn, Univ of Oregon Press, Eugene, OR.

15. Primavera JH (2009) Field Guide to Philippine Mangroves, Zoological Society of London, Philippines.

16. Schneider CA, Rasband WS, Eliceiri KW (2012) NIH Image to ImageJ: 25 years of image analysis. Nat Meth 9, 671-5.

17. Dong W, Macaulay LJ, Kwok KWH, Hinton DE, Ferguson PL, Stapleton HM (2014) The PBDE metabolite 6-OH-BDE 47 affects melanin pigmentation and THR $\beta$ MRNA expression in the eye of zebrafish embryos. Endocr Disruptors 2, e969072.

18. Chang $\mathrm{T}$ (2012) Natural melanogenesis inhibitors 
acting through the down-regulation of tyrosinase activity. Materials 5, 1661-85.

19. Bandaranayake WM (2002) Bioactivities, bioactive compounds and chemical constituents of mangrove plants. Wetland Ecol Manag 10, 421-52.

20. Harizon, Pujiastuti B, Kurnia D, Sumiarsa D, Shiono Y, Supratman U (2015) Antibacterial triterpenoids from the bark of Sonneratia alba (Lythraceae). Nat Prod Comm 10, 277-80.

21. Azizuddin, Khan AM, Choudhary MI (2011) Tyrosinase inhibitory potential of natural products isolated from various medicinal plants. Nat Prod Res 25, 750-3.

22. Herawati N, Firdaus (2013) 3,3'-di-O-methylellagic acid, an antioxidant phenolics compound from Sonneratia alba bark. J Nat Indones 15, 63-7.

23. Yoshimura M, Watanabe Y, Kasai K, Yamakoshi J, Koga $T$ (2005) Inhibitory effect of an ellagic acidrich pomegranate extract on tyrosinase activity and ultraviolet-induced pigmentation. Biosci Biotechnol Biochem 69, 2368-73.

24. Shimogaki H, Tanaka Y, Tamai H, Masuda M (2000) In vitro and in vivo evaluation of ellagic acid on melanogenesis inhibition. Int J Cosmet Sci 22, 291-303. 